<U34 TESPกU3U

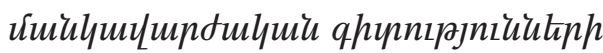

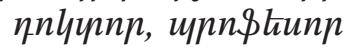

UUPQLPRSU UUPGप, U3UU

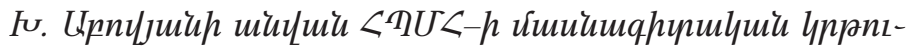

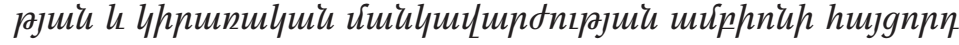

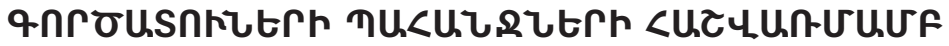

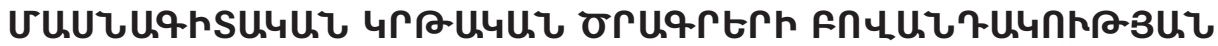

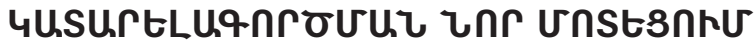

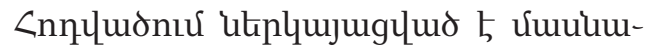

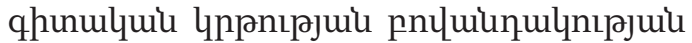

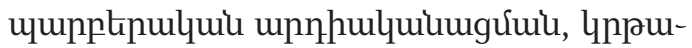

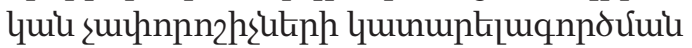

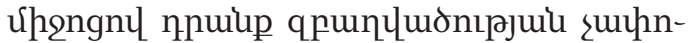

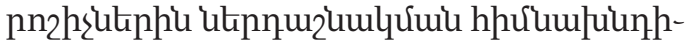

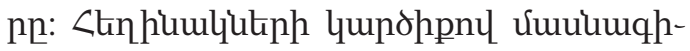

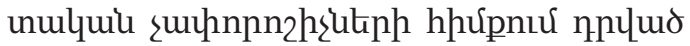
unuiggpujhì nnulquцnnnứutenn' 'utn

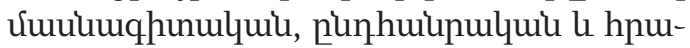
unum qnpownumlquil, npnzulh umulum-

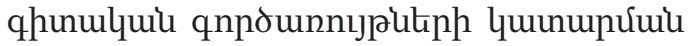

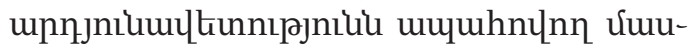

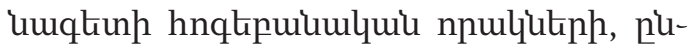

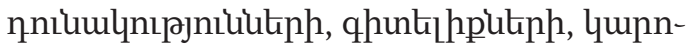

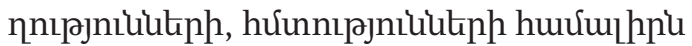

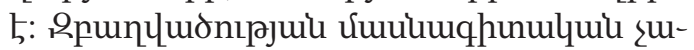

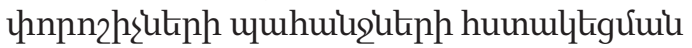

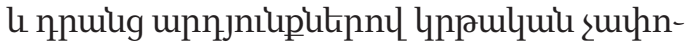

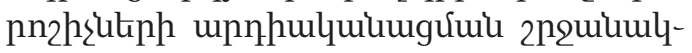
utipnux hpulquiumgltal 5 vuuumqhum-

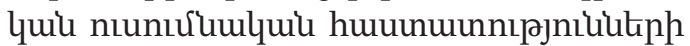
2nquiumumpulutphi utiplumugunn uщ-

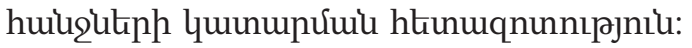
Chinuqnunnıрjuil upnjnilputinnl pugu-

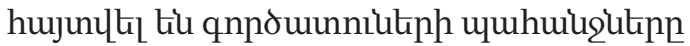

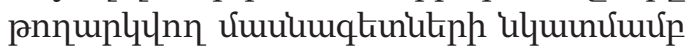

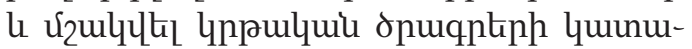

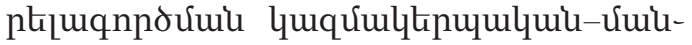

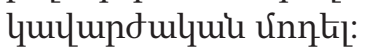

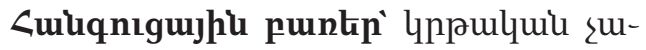

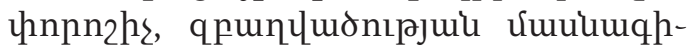

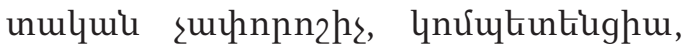

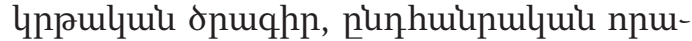

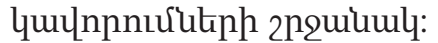

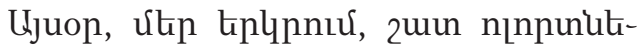

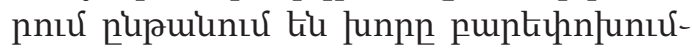
ukph qnnдpupuguten, nph htun quшцш

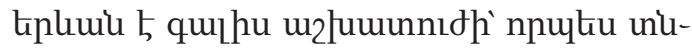
unkunıpjuil unuiggpujhu nkunıpup unp

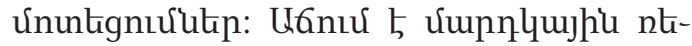
unınup ntipu unumunnnıрjuil utig unpu-

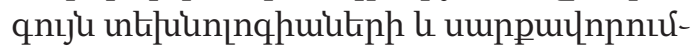

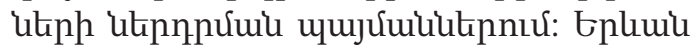
5 quihu unnumpnıрjuiu mpnjniuputpp

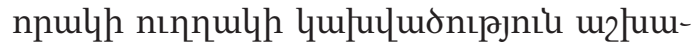

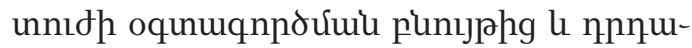
umunfunitiphg, umukuцnnumku tumb jnınupuilsjnın w2/uwunnnhg:

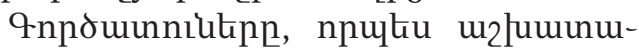

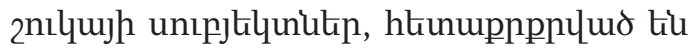
umulumqtinutiph pupughl umbuigupln

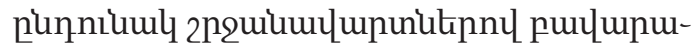
ntipnt hupgnu:

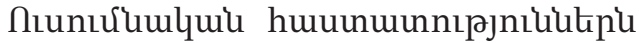
unultil hufaula ltiunnnumgur tiu ln-

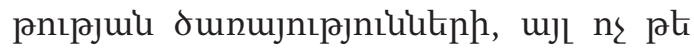

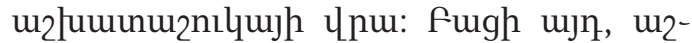

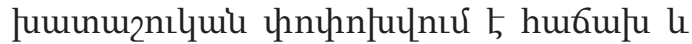

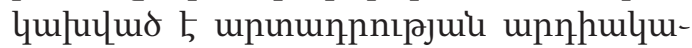

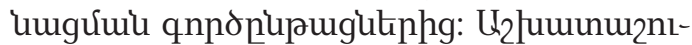
quil le 4npulquil dunujnıpjnilutiph 2nt-

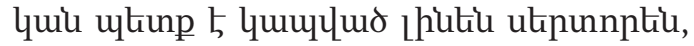
hul npuig enpuhupuptipnıpsniuutin

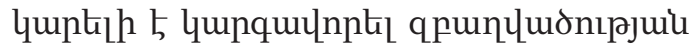

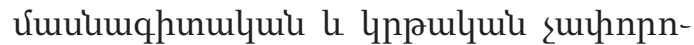




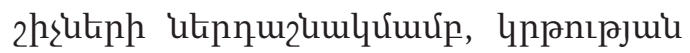
pnцuinulynıрjuiu umpptinuluiu qu-

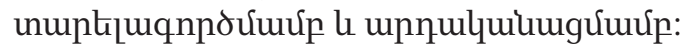

Uuuumqhumulqui lnpaulquiu suunnn-

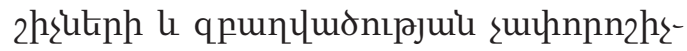

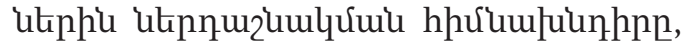

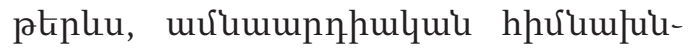

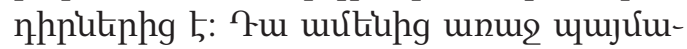

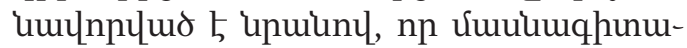

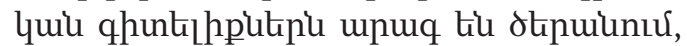

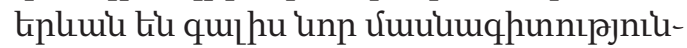

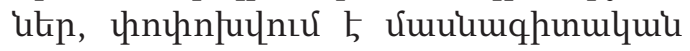

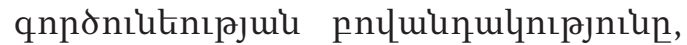
uhonghtipn le w2humunuipujhi umj-

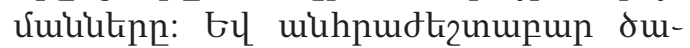

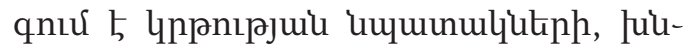
nhputinh, ulqpniuputiph umhर्umuर्uiu

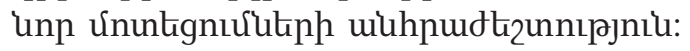

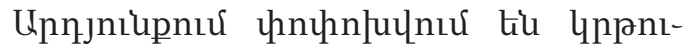

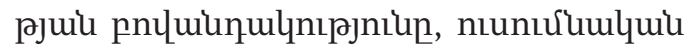

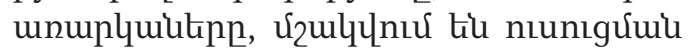

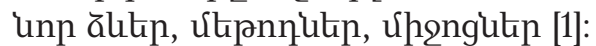

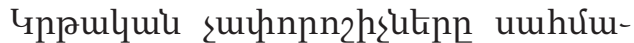

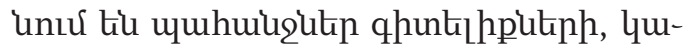

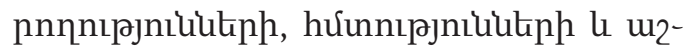

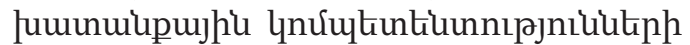

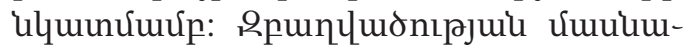
qhunuluu suuhnpnzhsutpn nnnzulh nןnpunnıर upununpnпuluiunıpjui $\mathrm{l}$ quinunцnn w2luminuiph npullh hw2-

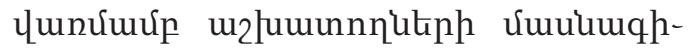

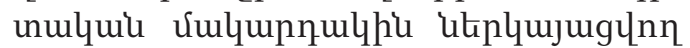

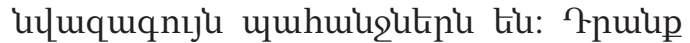

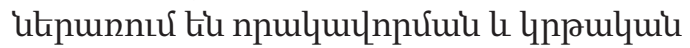

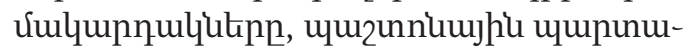

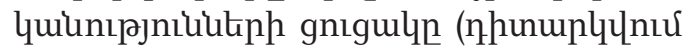
tiu qhunkhputiph, qunnnnıpsniuuknh,

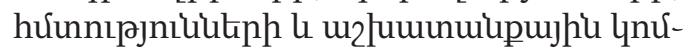

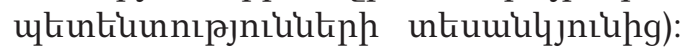

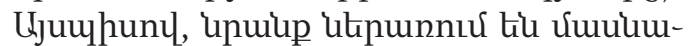

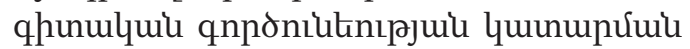
unpưunhl uwhwightinn: शpunцuonı-

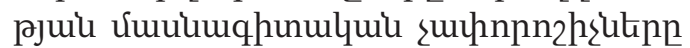
huunnıl quplinnnıpjnil tiu ătp ptipts

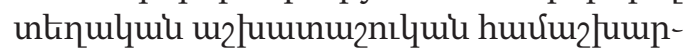

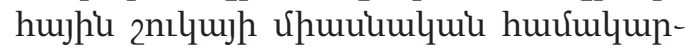

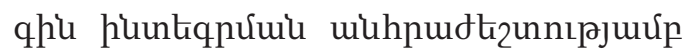

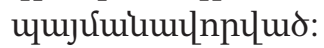

Ttin 2003p. U. 3nt. Slyušlu unmequnlthl

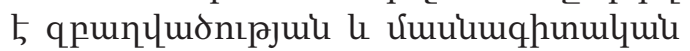

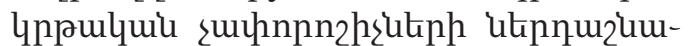

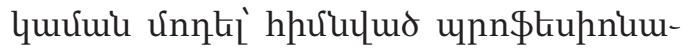
lhqư wlưtingnqhulquiu hnqtipuiumlumi

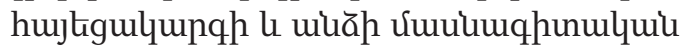

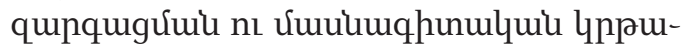
quiu úhquцujnh પnu [2]:

Uuuumqhunulyui lnpaulquiu suuhnnn2hrutepu nınпumo tiu úmulumqhunulquil unn\$tupniuul qnponiutnıрјuil шщш-

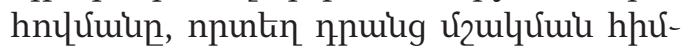
uwluiu pununphsitinn umulumqhumquil qhuntilhputiph, quinnnnıрлnıutitiph

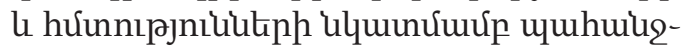
utpin tiù úmulumqting vouumuqhonu-

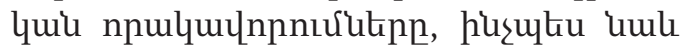

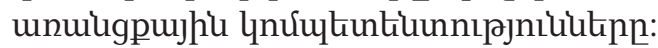

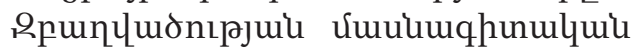

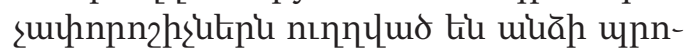

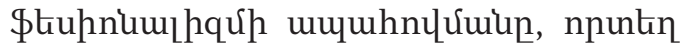

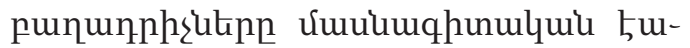
quik npuliktph qunqugúuin utiplum-

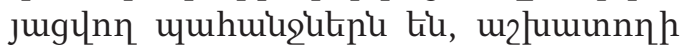

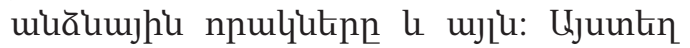
unulthl guplenp tiu unwugpujhu nnu-

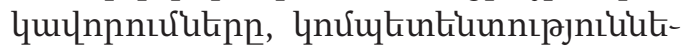

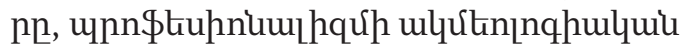
wünnhnjumlutunn:

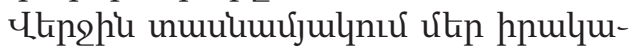

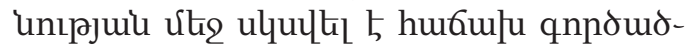

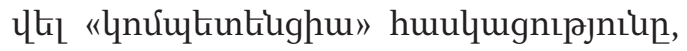

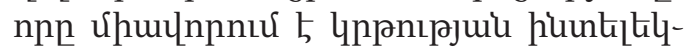

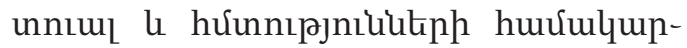

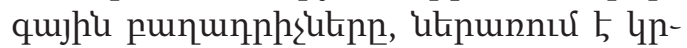

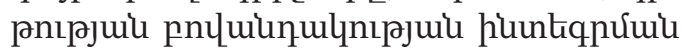

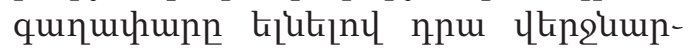

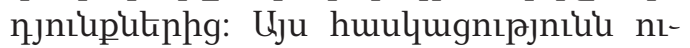
uh huuntqnumpl punujp: Utip lnnúug, ynưukintighuiu huulyugunıu 5 npuku

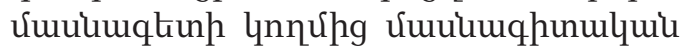
qnnoniuknıрjuiu dưưuiuly hp qhunk hp-

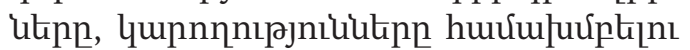

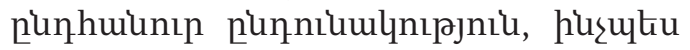




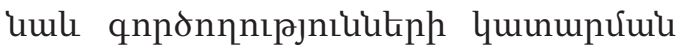
punhuiunugumo tnuiumlytun [1]:

Uuukuqhunulyui zuuhnnnzhiutiph

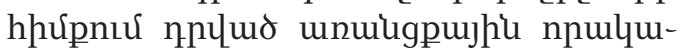

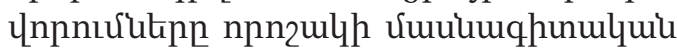

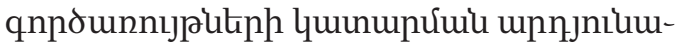

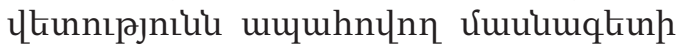
hnqtipuiumlquiu npuliukph, punniuwlyne-

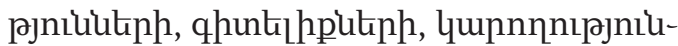

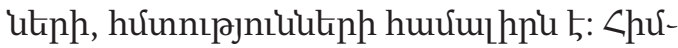

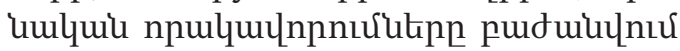
tiu tiplp lứph'

- 'utin úuuumqhumuluì vitl lnpulqui цuर्u qpunцuonıрjuiu úuuluwqhunnpjuiu hưưun huinnıl, hpuinuu,

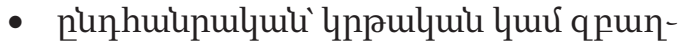
цuonıрjui ưuumuqhunnıjnilutiph juर्uph hưưu hpuinuw,

- hpuunuu qnpouniumluil guilqugur úmulumqhunuluiu úhquцuph hứup hpuinuuu:

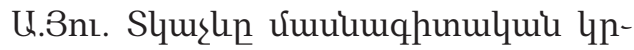

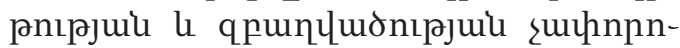

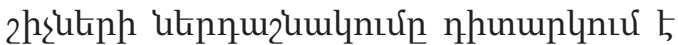

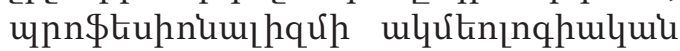

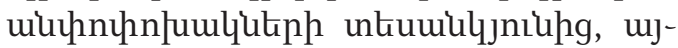
uhupu wulumu pnumunulynıpjniuhg $\mathrm{l}$

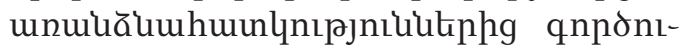

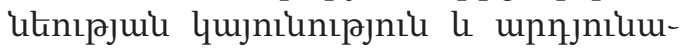

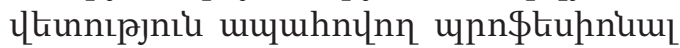

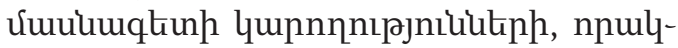
utnph, unumăumhumunnıpjniuutenh untuwulyjniuhg [2]:

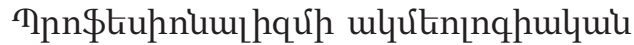

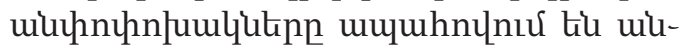

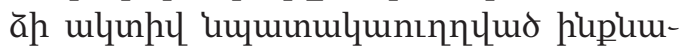
qupqugnıú, unknduqnnдuluuiu ut-

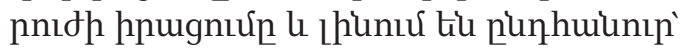

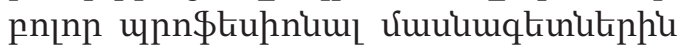
huunnıl, quर्u jnıpuhuunnıl' umuluwqhunulquiu qnnonititnıрjuil uwhuiqgitipu upunugnцnп:

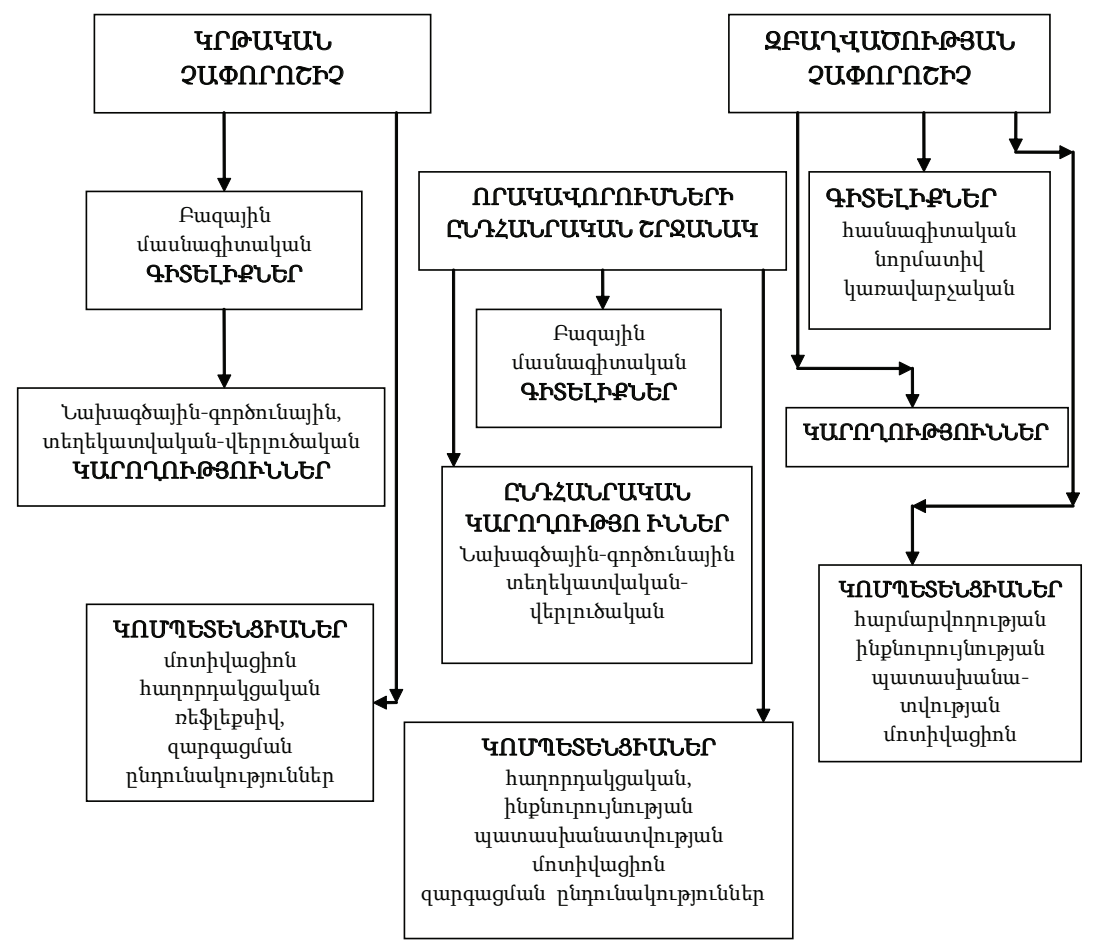

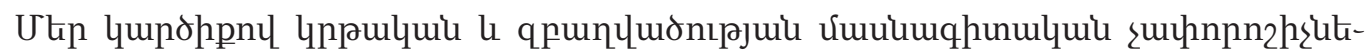

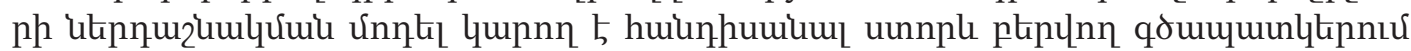

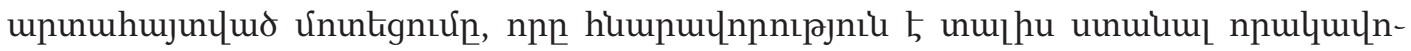


nnıরutuph punhuiunıр ulqupuqhnn npu-

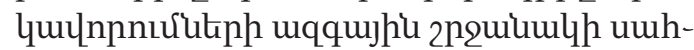
ưuuktinnıu:

Utip, ưưu unnutgưuiu hhưp 5 huiu-

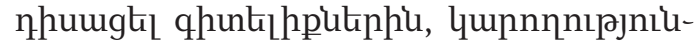

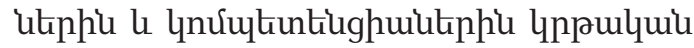
$\mathrm{l}$ qpunцmonıpjuil vuukuqhunulqui

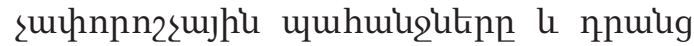

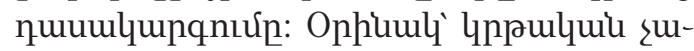

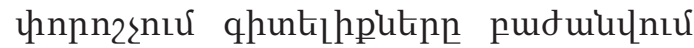
til puqujhlh $\mathrm{l}$ úmulumqhumulumh, hul qpunцuðnıpjuiu vuuumqhumulumi $2 \mathrm{w}-$

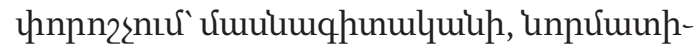

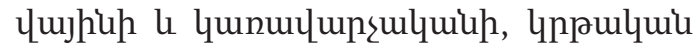

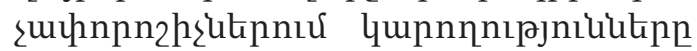
puruiluntu til umpumqdujhu-qnpont-

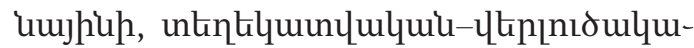
uh, hul qpunцmonıpjuil umulumqhunu-

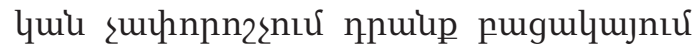

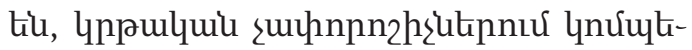
untiughuutipu uúpnngugunıu tiu unnonumghnu, hunnpnulgumuil, ntipltipuhl

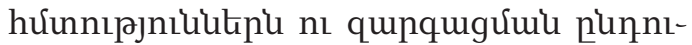
uulynıpjniuutens, hul qpunцuðnıрjui

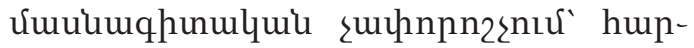
রunцnnulquiunıpjnıนn, hupunıpnıjunıpjnikn, umunuuluwumunцnıpjnikn, únunhumghuiu:

Gnulphin, ophumb umuimqhonulyuiu qhunkihputiph, nhonuplưum duर्umiuml hunuly unumitilh tiu ujn qhunthplitnp le npuiug puqplynn njnpuituph pun-

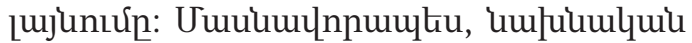
úuuumqhunulyuu (uphtiunuqnpoulquiu)

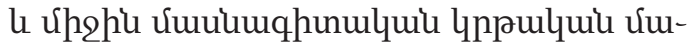

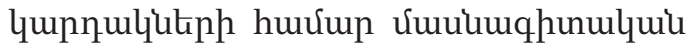

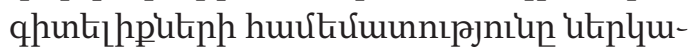
juglud b unnplu ptipunn unjnıumlynứ:

\begin{tabular}{|c|c|c|c|c|}
\hline \multirow{2}{*}{ 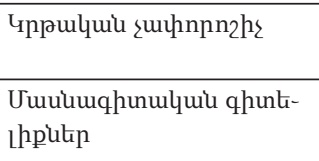 } & \multicolumn{3}{|c|}{ 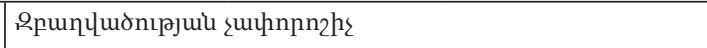 } & \multirow{2}{*}{ 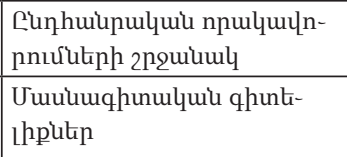 } \\
\hline & $\begin{array}{l}\text { Uuukumqhunulquil } \\
\text { qhuntihputin }\end{array}$ & $\begin{array}{l}\text { Gnpưuunhl } \\
\text { qhuntilhputep }\end{array}$ & $\begin{array}{l}\text { 4unulunsulqui } \\
\text { qhunthputip }\end{array}$ & \\
\hline 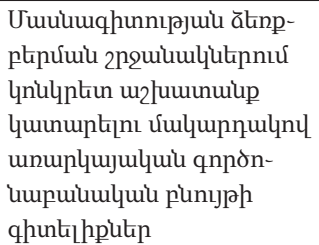 & 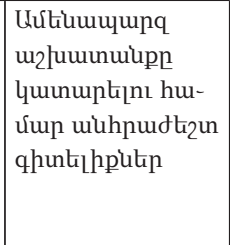 & $\begin{array}{l}\text { U}_{2} \text { luwumuipujhi } \\
\text { hpuhuiuqutph } \\
\text { hưugnıpniu }\end{array}$ & $\begin{array}{l}\text { 9hunthputp wi- } \\
\text { hpudtžn ¿̨lu }\end{array}$ & 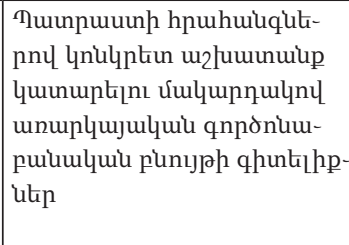 \\
\hline 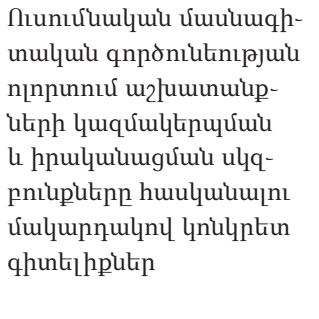 & 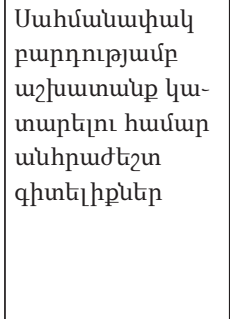 & 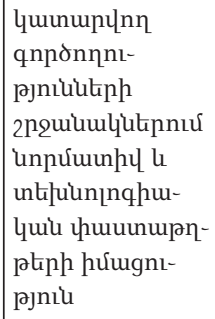 & 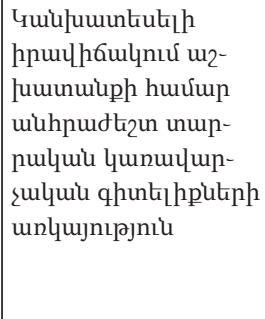 & 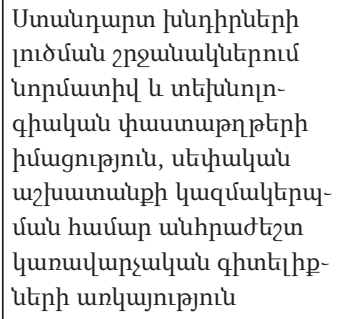 \\
\hline 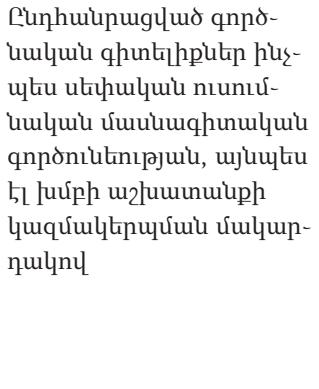 & 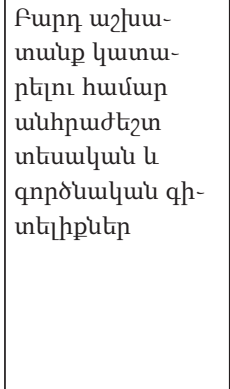 & 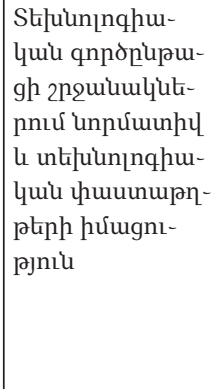 & 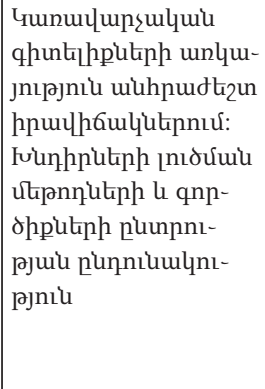 & 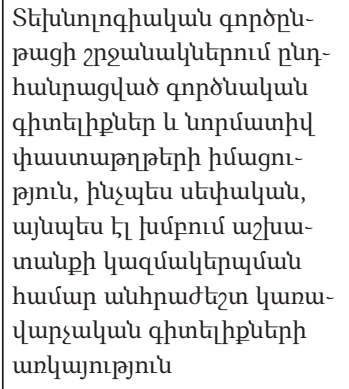 \\
\hline
\end{tabular}


Uuulumqhunuluiu qhunklhputph ưuqupnulikinn htinuqujnı qupnn tiu

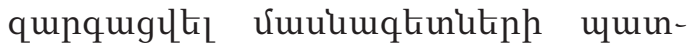

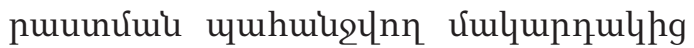

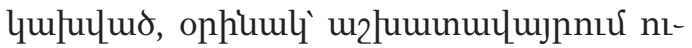
unıgर्uil duर्umuml, दnpunnumhl b ujl

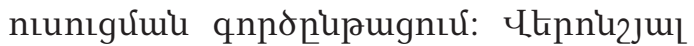

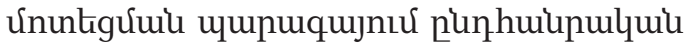

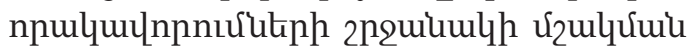
qnpopipugnı unp humpuцnnnıpjniuutip tiu uuknoulnuर '

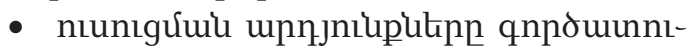
ukph uquhuiqukiphi hưưuqumuajumilitgúuiu,

- zumnnnzhritipniu umhuiqutiph ulymuqnúuil úpuukumuil unkumi4Jniuitinhe,

- quuhuunumu lu huцluunuqpúuiu hu-

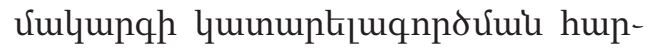
gknnuर:

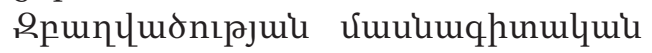

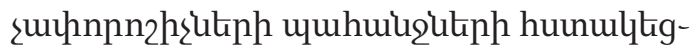

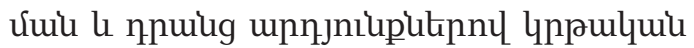

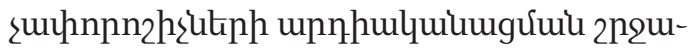

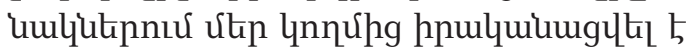

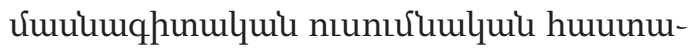
unnıjniukteph 2nquiumumpuntephi uten-

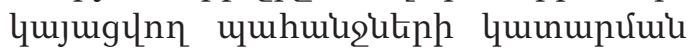
htinuqnunnıpjnil: <unguqpnıgh lu wiulkinulnpưulu úhongny qnpouunniukph

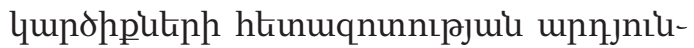

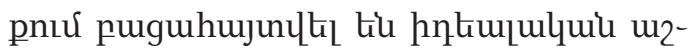

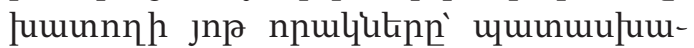

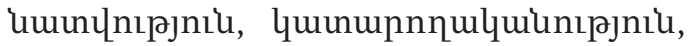

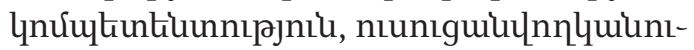

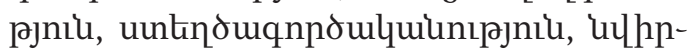

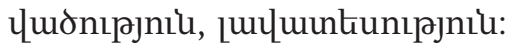

Zuin qnnдuunnilitph цunßhpnц (23\%)

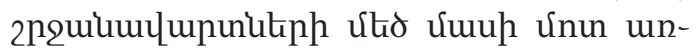
qu tiu juluqnuju zwin npulyktip: huluuku, uјuonцu kphunuump ukpniknp

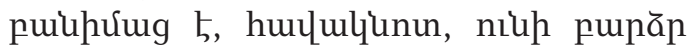
puuteltilun, qupnnuiunıu 5 oqunuqnn-

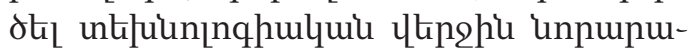

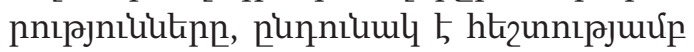

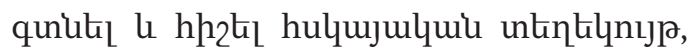

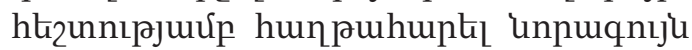
unthun_nqhuitinn: Uhuduruuuml, qnnduunniutipn (19\%) uznou tiu úuuumqhunuquil qhunkihputiph úulkpkumjunıрjui,

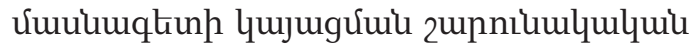

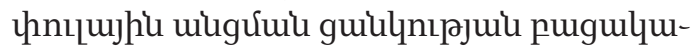

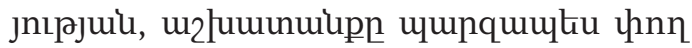

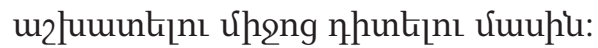

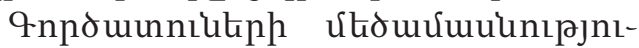

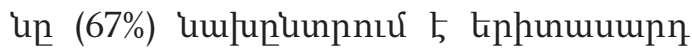
2nquiumumunutiphi unuigg b'uknqhuiu, ulqunццnıрjniun, zwndniunıpjniun, hul

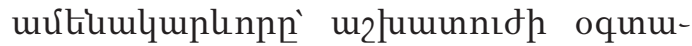

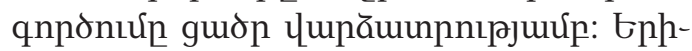

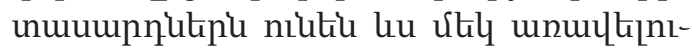
pjnil, upuip wutilh htizu tiu hiunkqnunuर

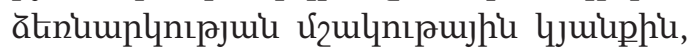

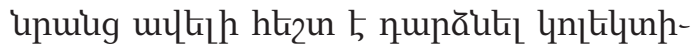

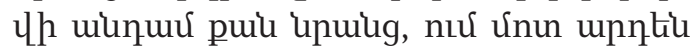

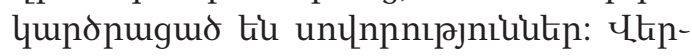

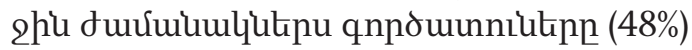

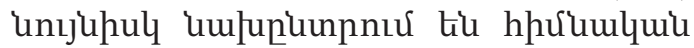
umulumqhunulquiu qhuntihputen sniltignn,

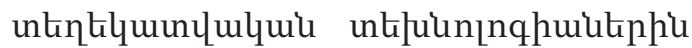

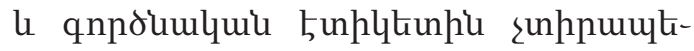
unn tiphunuuwpntiph, humpuunn hu-

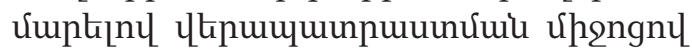

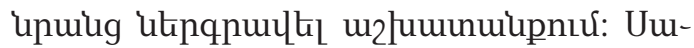

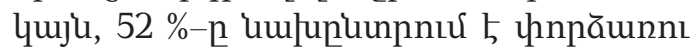

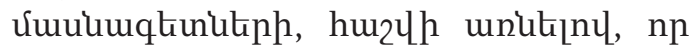

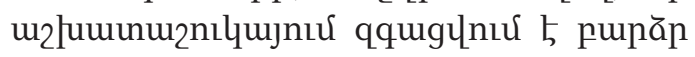

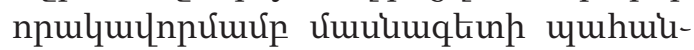
¿upl l quiunúu b, np tiphunuump 2nquumulupuntiph únu pugumujnıu tiu lum-

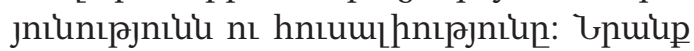

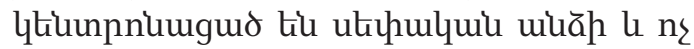

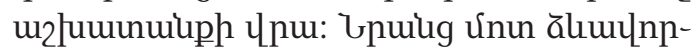
цuo 5 unp undthưumlqunq:

Builqugur qnpouinnt 2nquiumulupn-

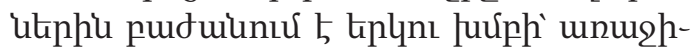

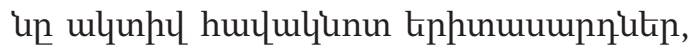

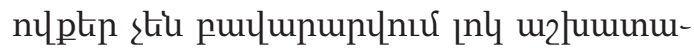

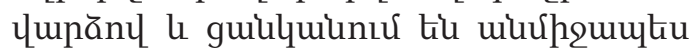
puguhujunts ukihulyuia punnitumlynt- 


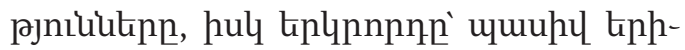
unuumpnitin, npnup puцupunцnuर tiu

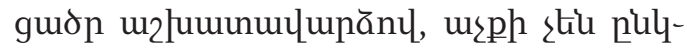

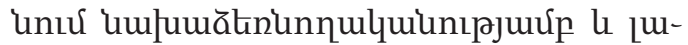

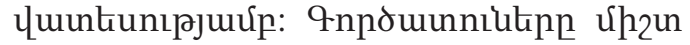
25, np puonnnuर tiu 2nquiumumunutiph unwęh buúphg: ঢnuip 2nquiumump-

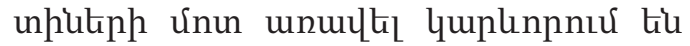

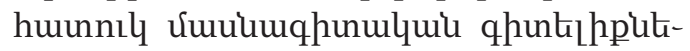

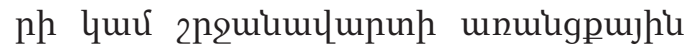

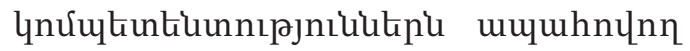

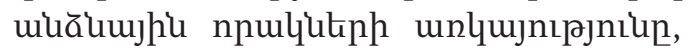

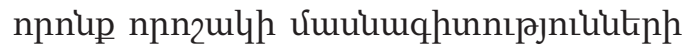

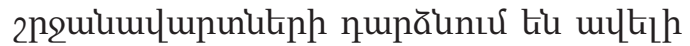
ungnituwl:

Ujuuhunц, hnulquiumgluo hunguq-

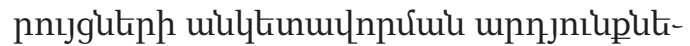

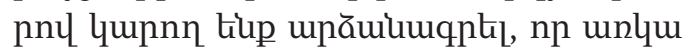

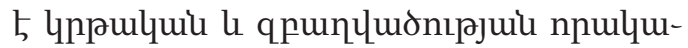

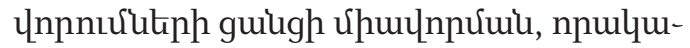

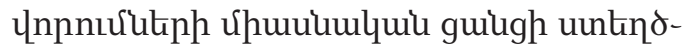
ưu uuhnudtizunnıرnilu: Ujn guugh hhúpnuर्u ukinp 5 nnцtiu qhuntilpputiphu,

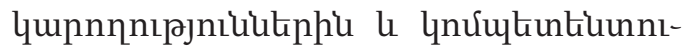

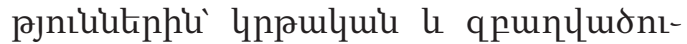

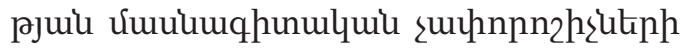

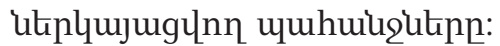

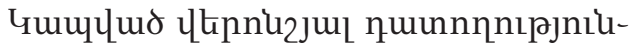

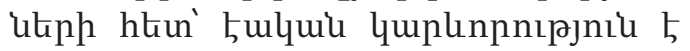

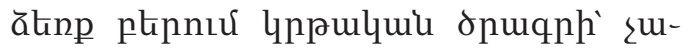

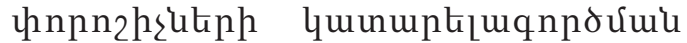

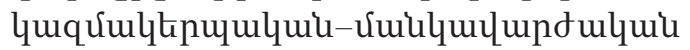

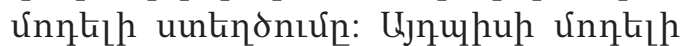
úmlyर्umu hưưp niktiumpl htionuqn-

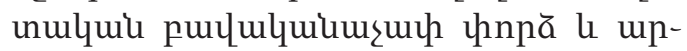

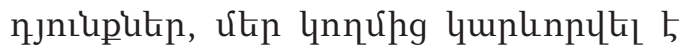

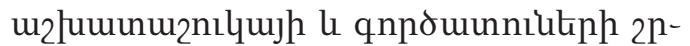

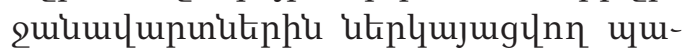

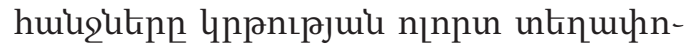
jutinı hungh jnıðnıún:

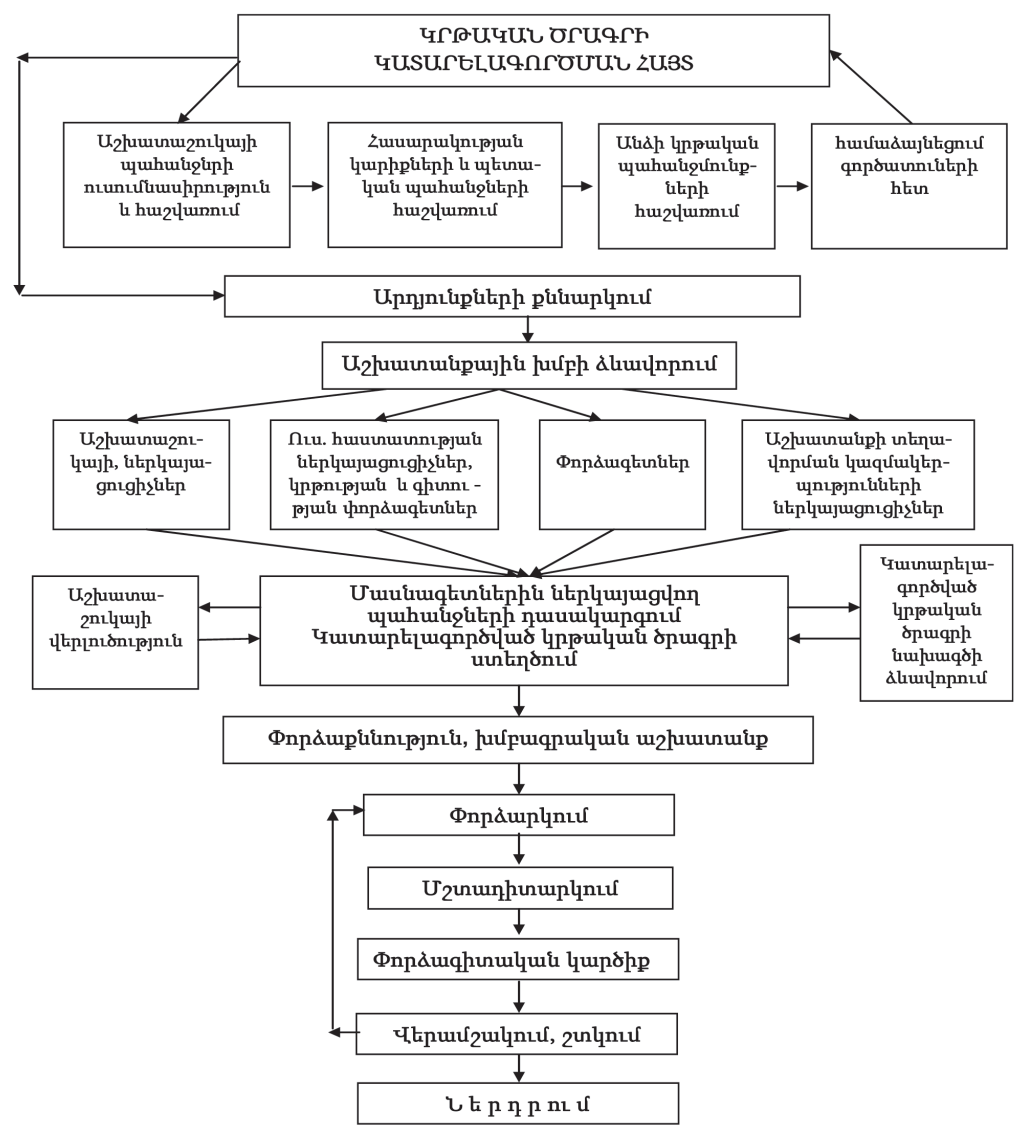




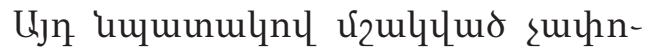
nnzhsikph quinupkjuqnnoर्umu quq-

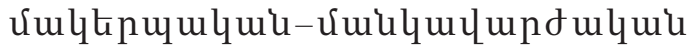

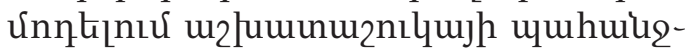
ukph nıunıর'umuppưul $\mathrm{l}$ hurlunumil, huumpulpnıрjuil quphpitiph,

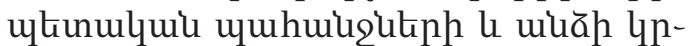

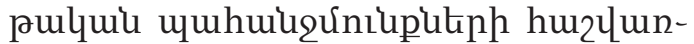
ưul hưưunkpuunnu nıpnıju unkn 5 huunlugglti qnpowunniktiph htiun huưmămukgưuil pusıhu:

Uhudurumiuml, w2 fumunurnılyujh

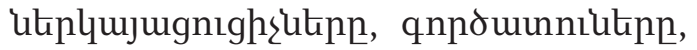

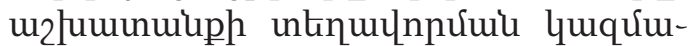
linunıрлniuutiph utplqujugnighsutinn utenqnulцuo tiu lnpulquil opuqpiph

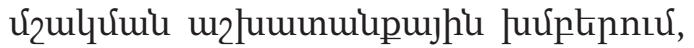
úuukuqtinutiphi ukplqujugunn uш-

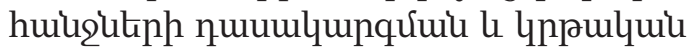

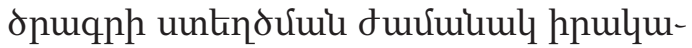

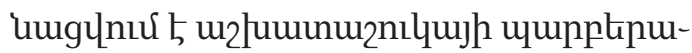

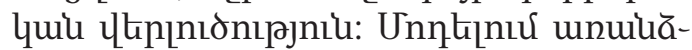
uuhuunnil quplennlta 5 úmulumo

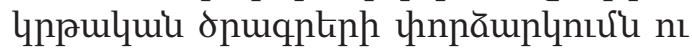

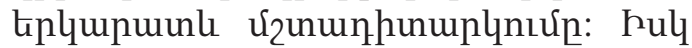

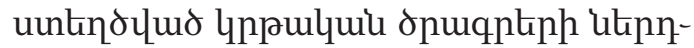
ưuun uulunpnnıu 5 unnăuqhunuluuu

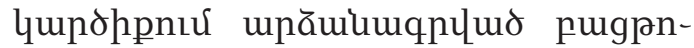

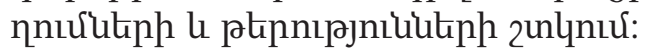

Ujuuhunl, 4npulqui opuqnten

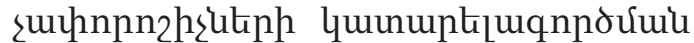

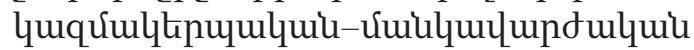
uuntndy ư unntęn huwnuцnnnıрjniu lpuătinh qnnduunntuknp u2fumunu2nılqujh umbeigitipis upunuhujunts

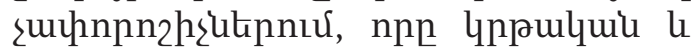

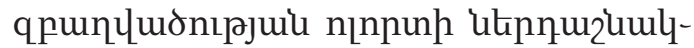
ưuu nınhn le hnıuuilh úhonglu 5 :

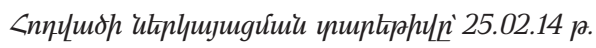

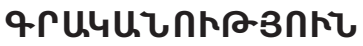

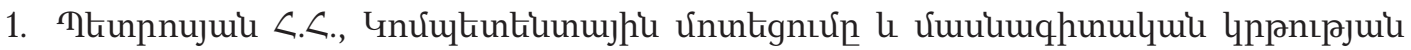

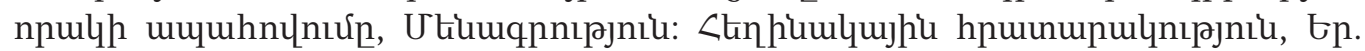
2013.- 264 52:

2. Ткачев Ю.А. Образовательные и профессиональные стандарты: поиск теоретикометодологических оснований // Психология профессионально-образовательного пространства личности: сб. науч. ст. - Екатеринбург, 2003. - С. 142-148.

\section{СОВЕРШЕНСТВОВАНИЕ СОДЕРЖАНИЯ ПРОФЕССИОНАЛЬНЫХ ОБРАЗОВАТЕЛЬНЫХ ПРОГРАММ С УЧЕТОМ ТРЕБОВАНИИ РАБОТОДАТЕЛЕЙ}

\section{ПЕТРОСЯН ГАЙК}

\author{
Доктор педагогических наук, профессор
}

\section{МАТЕВОСЯН МАРГАРИТА}

\section{Соискатель кафедры профессионального образовавия и приклодной педагогики АГПУ имени Х. Абовяна}

В статье представлены проблемы периодического обновления содержания профессионального образования, путем совершенствования образовательных стандартов, их гармонизации стандартам занятости. По мнению авторов, ключевые квалификации, лежащие в основе профессиональных стандартов (узкопрофессиональные, обобщенные, актуально-функциональные),- это комплекс психологических качеств, 
способностей, знаний, умений, навыков специалиста, обеспечивающий эффективное выполнение конкретных профессиональных функций. В рамках уточнения требований профессиональных стандартов занятости и по их результатам модернизации образовательных стандартов были исследованы требования к выпускникам профессиональных учебных заведений. В результате исследования были раскрыты требования работодателей к подготовке специалистов и разработана организационнопедагогическая модель обновления образовательных программ.

Дата представления статьи: 25.02 .14 г.

\title{
NEW APPROACH TO IMPROVING EDUCATIONAL PROGRAM CONTENT TAKING INTO CONSIDERDATION EMPLOYERS’ REQUIREMENTS
}

\section{HAYK PETROSYAN}

\author{
Doctor of Pedagogical Sciences, Professor
}

\section{MARGARITA MATEVOSYAN}

\author{
Applicant for PhD Degree at the Chair of Professional Education and Applied \\ Pedagogy, Armenian State Pedagogical University after Kh. Abovyan
}

The article presents the issue of regular updating professional education content through improving educational standards and harmonizing them with employment standards. According to the authors, key qualifications serve the basis of professional standards (narrow professional, generalized, urgently functional), a complex of psychological qualities, abilities, knowledge, competences and skills of professionals ensuring effective implementation of certain professional functions. Requirements to the graduates of vocational schools have been studied in the frames of clarifying requirements and professional standards of employment on the results of modernizing educational standards. The results of the studies identify employers' requirements to graduates, afterwards an organizational-pedagogical model for improving and ameliorating educational programs have been developed. 